\title{
流动量与关注度视角下的城市网络结构 以广州、深圳为例
}

\author{
吴 炫, 杨家文* \\ (北京大学深圳研究生院城市规划与设计学院,广东 深圳 518055)
}

\begin{abstract}
摘 要: 在城市与区域转型发展的背景下, 城市网络经历着剧烈的重构,广州和深圳是推动粤港澳大湾区一体化的 主导力量, 明晰其在网络中的发展定位与动态联系, 对于引领区域协调发展具有重要的战略意义。然而, 现有城市 网络研究较缺乏对多尺度差异与实虚映射关系的关注, 因此论文基于微博数据, 从流动量、关注度出发, 运用社会 网络分析,探究了广深在粤港澳大湾区、全国、全球网络中的节点地位与联系特征。结果表明: (1) 多尺度网络下, 广深不同的联系导向塑造了差异化的要素组织能力, 广州辐射范围较广、联系相对均衡, 深圳联系相对集中、与香 港联系尤为紧密; (2) 实虚网络之间,广深的对外联系存在协同补充、路径依赖效应, 且在各尺度下具有不同程度的 体现, 多重效应的叠加交融推动着区域联系趋向柔性化; (3) 基于上述网络格局,广深应立足于不同的联系模式与 发展实际, 分别发挥区域交通枢纽及创新制度高地的优势,引领打造有序高效的区域网络系统。
\end{abstract}

关 键 词: 城市网络; 微博; 流动量; 关注度; 粤港澳大湾区

在城市与区域转型发展的背景下, 随着跨域要 素流动规模的不断增长, 区域一体化的空间发展框 架已逐渐拉开(林雄斌等, 2015)。地理学者先后开 创了世界城市假说(Friedmann, 1986)与全球城市理 论(Sassen, 1991), 认为城市间的经济活动构筑了复 杂的层次结构, 部分关键城市已成为全球资本积累 的重要节点。之后, Castells(1996) 在网络社会理论 中提出了 “流空间” 的概念, 指出城市发展并非依托 本地化的静态功能, 而是通过网络交互联系塑造了 不同的区域发展地位与资源组织能力。在此基础 上, 社会网络分析等理论框架相继被引人城市区域 系统研究, 以进一步测度、解释跨域要素的流动、作 用及演化过程, 网络化由此成为了一项重要的组织 原则(聂晶金金等, 2017)。

粤港澳大湾区(简称 “湾区”)是中国参与全球竞 争的重要空间载体, 呈现出多核共生的城市网络格 局。而中心城市作为区域网络体系的组织节点, 其 互动联系是整合跨域要素、推动区域增长的核心动
力(姚永玲等, 2015)。广州、深圳同属粤港澳大湾 区, 作为引领湾区一体化的主导力量, 两者在人口、 GDP 等传统静态发展特征上具有高度相似性(广州 市统计局, 2017; 深圳市统计局, 2017), 但由于联系 结构、发展路径等原因, 两市在湾区内外动态联系 上存在着较大差异。因此, 以广州、深圳为例, 明晰 二者在区域网络中的发展定位与互动关系, 对于支 撑国家空间战略的推进、探索谋划区域协调发展模 式具有重要的战略意义。

此外,在信息技术的影响下,城市网络经历着 剧烈的动态重构, “流空间” 展现出了更加丰富的内 涵, “距离死亡论”(Cairncross, 2001)引起了学界的 广泛讨论。当前, 静态分布、时效滞后的传统数据 已难以反映出日益复杂的联系格局, 而近年来涌现 出的带有地理标签、移动轨迹的 “流”数据则为城市 网络研究提供了海量数据支持(柯文前等, 2015)。 国内外学者通过 Twitter、Flickr、微博、百度指数等 数据, 刻画了人口流动、边界划定、意向感知、出行

收稿日期 : 2019-01-02 ; 修订日期 : 2019-03-19。

基金项目: 国家自然科学基金项目(51678004)。[Foundation: National Natural Science Foundation of China, No. 51678004. ]

第一作者简介: 吴炫(1995-), 男,福建晋江人, 硕士生, 研究方向为城市区域交通。E-mail: 470449189@qq.com

*通信作者简介: 杨家文(1974-), 男, 湖南东安人, 教授,博士生导师, 研究方向为城市区域交通。E-mail: yangjw@pkusz.edu.cn 
驱动等城市动态交互格局(董超, 2012; Belyi et al, 2017; Yin et al, 2017; 谢永俊等, 2017)。

然而, 纵向视角下, 现有城市网络研究侧重于 揭示单层级网络的整体结构特征, 缺乏对个体节点 在不同尺度网络下联系差异的关注; 多要素横向对 比上, 学界多采用多源数据, 在实虚映射关系的可 比性、潜在偏差等方面易引起质疑。因此, 本文拟 回答 2 个重要问题: (1) 多尺度网络下, 广州、深圳分 别展现出了什么特征, 不同尺度间存在何种差异? (2) 实虚网络之间,如何理解广深联系的映射关系,2 类联系经由哪些路径相互影响? 基于以上认知, 本 文以新浪微博 API 单个数据源, 通过实体跨城流 动、虚拟异地关注 2 个视角, 从湾区、全国、全球 3 个 尺度构建有向加权城市网络, 尝试剖析广州、深圳 的节点特征与联系差异, 进一步透视 “流空间”理论 框架下城市网络的作用形态、演进路径及区域响应 (王萌等, 2017), 以期为构建广深竞合背景下的空间 发展战略与区域发展体系提供理论依据。

\section{1 数据来源与研究方法}

\section{1 数据来源}

本文通过新浪微博API 获取了自 2018 年 1 月 1 日-2月 6 日共 $37 \mathrm{~d}$ 的数据, 研究时段避开了春运 时期, 经过清洗、去重后, 得到 10697995 条带有地理 位置的微博。按时间整合用户流动链, 可得 $\left\{\left(l_{1}, t_{1}\right)\right.$, $\left.\left(l_{2}, t_{2}\right), \cdots\left(l_{n}, t_{n}\right)\right\}, l_{i}$ 和 $t_{i}$ 分别表示第 $i$ 次签到的位置与 时间, 并移除连续签到间移速超过 $1000 \mathrm{~km} / \mathrm{h}$ 的异 常数据(Hawelka et al, 2014), 进一步计算“流数据” 的 2 类替代参量。

(1) 流动量: 表征城市之间的实体人流联系次 数。根据用户流动链, 比较连续 2 次签到位置, 若城 市不同, 则判定 2 城间发生了一次跨城流动, 反之则 未发生流动。

(2) 关注度: 表征城市之间的虚拟信息流联系 次数。以签到位置为城市 $i$, 辅以过滤条件, 若微博 文本中检索到城市 $j$ 名称, 则判定为城市 $i$ 对 $j$ 的一 次异地关注(全球网络中, 以英文大小写、首字母大 写及中文名集合进行检索)。

不同尺度下, 网络联系的表征内涵与作用机制 具有差异, 因此将 2 类替代参量贯穿 3 个尺度, 分别 除以该尺度网络中的最大值, 以获取相对值。研究 结果与珠三角(谢永俊等, 2017)、中国(王姣娥等,
2017)、全球网络(Choi et al, 2006)等视角下的联系 格局相近,一定程度上印证了数据的有效性。

\section{2 研究方法}

\subsection{1 社会网络分析}

以城市为节点, 以流动量、关注度为边权重, 分 别构建有向加权网络, 并综合运用Matlab、Gephi 等 软件对网络格局进行分析。

（1）度中心性(Alderson et al, 2004): 衡量节点在 网络中的地位及影响力, 即节点连接的所有边权重 之和。公式如下:

$$
C_{D}(i)=\sum_{j} w_{i j}
$$

式中: $C_{D}(i)$ 为度中心性, $w_{i j}$ 为 $i$ 到 $j$ 的边权重。本 文中, $C_{D}(i)$ 取出、人度中心性之和。

(2) 接近中心性(Alderson et al, 2004): 衡量节点 在网络中的通达性程度,即节点至其他可到达节点 距离之和的倒数(以边权重的倒数作为边长度)。公 式如下:

$$
C_{c}(i)=\left(\frac{A_{i}}{N-1}\right)^{2} \frac{1}{D_{i}}
$$

式中: $C_{c}(i)$ 为接近中心性, $A_{i}$ 是节点 $i$ 可到达的节点 数, $N$ 是网络中的节点总数, $D_{i}$ 是从节点 $i$ 到所有可 到达节点的距离之和。本文中, $C_{c}(i)$ 取出、入接近 中心性的平均值。

(3) 首、次位联系(王姣娥等, 2017): 指与广深的 双向边权重之和位于节点对外交流第一、二位的城 市联系, 反映了该节点的跨域要素流动在一定程度 上受到广深的组织配置。

为确保可视化分析的准确性, 依据最大化维持 信息相关性、丰富度的原则进行敏感性检验(王永 明等, 2012), 以相关系数、香农熵绘制碎石图(Scree Plot), 找到特征转折点作为划分联系层级的阈值, 通过网络过滤进一步简化、概括网络特征。

\subsection{2 平均旋转半径}

平均旋转半径代表居民流动行为的空间范围, 其值越高表示流动范围越大。其中, 判断用户居住 地的规则为: (1) 19:00 6:00内, 取签到频数最高的 城市为居住地 (Xu et al, 2018); (2) 若该时间段内无 签到, 取签到总频数最高的城市为居住地; (3) 若多 个城市频数相同, 取距离签到重心最近的签到城市 作为居住地。平均旋转半径公式如下:

$$
R_{g}=\sqrt{\frac{\sum_{i=1}^{n}\left(\vec{l}_{i}-\vec{l}_{c}\right)^{2}}{n}}
$$


式中: $\vec{l}_{i}$ 为位置向量, $\vec{l}_{c}=\sum \vec{l}_{i} / n$ 为签到重心, $R_{g}$ 为 旋转半径。

\section{2 湾区城市网络下的广深对比分析}

粤港澳大湾区尺度下, 本文选取广东省 9 个地 级市及香港、澳门 2 个特别行政区为网络节点, 研究 时段内共有 861862 条湾区签到微博, 其中, 广深微 博分别占 $31.93 \% 、 30.42 \%$ 。

以节点特征来看, 广州在流动网络中优于深 圳, 度中心性高达 15153(表 1)。度中心性从集计层 面反映了网络联系决策结果, 侧重于刻画节点控制 力及区位禀赋, 广州作为湾区交通枢纽、政治经济 中心, 展现出了广阔密集的外向流动关联, 以实现 经济活动、市场空间与网络运转相匹配。另一方 面, 流动接近中心性则主要度量网络连接性和联系 效率, 广州坐落于连接东西岸的交通要道上, 铁路、 公路交通体系积厚成势, 接近中心性为 47.39 , 居湾 区首位, 显示出了良好的网络通达性, 地方场所的 邻近使其便于深人湾区城市、高效对接腹地, 进而 整合吸纳资源。联系导向方面, 广州与东、西岸城 市的联系相对均衡, 分别占其对外流动量的 $57.92 \% 、 42.08 \%$ 。联系广度方面, 平均旋转半径达 $87 \mathrm{~km}$, 首、次位联系可辐射湾区全域, 但目前仍局 限于近域拓展, 其与网络边缘的惠州、肇庆、江门三 地的联系总量仅占对外流动量、关注度的 $11.39 \%$ 、 $7.91 \%$, 对于潜在腹地的纵深关联、引领带动仍有较 大发展空间。

深圳则在关注网络中具有显著优势, 度中心性 为 5185 , 较广州高出 584 。深圳作为湾区信息枢纽 与创新高地, 随着信息需求、产出与消费的迅速增 长, 其隐性知识的外溢效应推动了湾区关注网络的 演化, 也使得个体对于地方场所的主观认知水平获 得了提升。另一方面, 关注接近中心性体现为城市

\section{表 1 湾区城市网络指标}

Tab.1 Indices of the Guangdong-Hong Kong-Macao Greater Bay Area's city network

\begin{tabular}{cccccc}
\hline \multirow{2}{*}{ 指标 } & \multicolumn{2}{c}{ 流动网络 } & & \multicolumn{2}{c}{ 关注网络 } \\
\cline { 2 - 3 } \cline { 5 - 6 } \cline { 5 - 6 } 广州 & 深圳 & & 广州 & 深圳 \\
\hline 度中心性 & 15153 & 14605 & & 4601 & 5185 \\
接近中心性 & 47.39 & 45.76 & & 11.89 & 12.27 \\
首位联系 & 5 & 3 & & 3 & 3 \\
次位联系 & 3 & 1 & & 2 & 4 \\
\hline
\end{tabular}

感知下形成的情境约束, 深圳与香港地理接近、制 度接近等因素成为了网络发育初期的推动力, 接近 中心性高达 12.27 , 较低的信息流转成本使深圳拥 有了更多直接参与、嵌人网络的机会, 进而以网络 延伸带动信息技术的全域化发展(孙中伟等, 2009)。但以流动网络来看, 深圳地处珠江东岸, 受 空间距离、水体屏障制约,联系能力弱于广州, 度中 心性仅为 14605 。联系导向方面, 深圳的对外联系 相对集中, 港深互动联系占深圳对外流动量的 $41.50 \%$, 但与西岸城市流动量的占比之和却不足 $15 \%$, 呈现极化现象。联系广度方面, 深圳的平均 旋转半径为 $56 \mathrm{~km}$, 辐射范围小于广州, 与莞、惠、港 形成了 3 对首位联系, 主要依托东进战略带动深莞 惠一体化建设,但拓展西岸腹地具有一定难度。

为了全面理解网络联系格局, 经敏感性检验、 网络过滤后, 根据碎石图的特征转折点将联系划分 为主干线、次干线、支线 3 个层级, 并以城市的度中 心性表示节点大小(图 1)。

以联系格局来看,深港间的人流、信息流互动 联系占据前列(表 2)。流动网络中,两地的频繁互动 与其产业合作的发展需求有关,改革开放初期, 港 商依托人缘地缘优势, 以深圳为窗口建立了“前店 后厂”的经济分工格局; 如今随着前海蛇口自贸区、 河套创新合作区的加速开发，两地金融、交通、贸易 等领域的合作不断深化, 深港合力发展的潜能将进 一步释放。关注网络中, “深一港”单向关注度高达 1601 , 位居首位, 这主要是由于深圳在引人香港的 国际市场信息、创新制度技术等战略实施中更加主 动作为。但另一方面, 反向的“港一深”关注度则骤 减至 550, 呈现联系断层现象: 一是可能缘于两地微 博用户覆盖率的差异, 香港签到微博仅占湾区的 $7.01 \%$, 关注主体的活跃性稍弱; 二是文化、制度性 障碍影响了城市主体的联系导向(张林等, 2006), 关 注认同感尚待提升。

其次, 广深、广佛联系也是支撑湾区网络的主 骨架。广州联系通道完备、经济腹地广阔, 深圳则 在创新生态体系、资本输出引人上占优, 两者间流 动量、关注度相对值介于 $0.45 \sim 0.77$, 位序均处前列, 两者以多层次、多领域的联动竞合引领着湾区网络 格局的更迭; 而广佛两地市区在空间上邻近, 流动 量相对值达 0.66 以上, 交通运输的接驳与公共服务 的统筹在一定程度上推动了同城化发展,但其信息 联系发展不足, 关注度相对值均低于 0.26 。 
(a) 流动网络

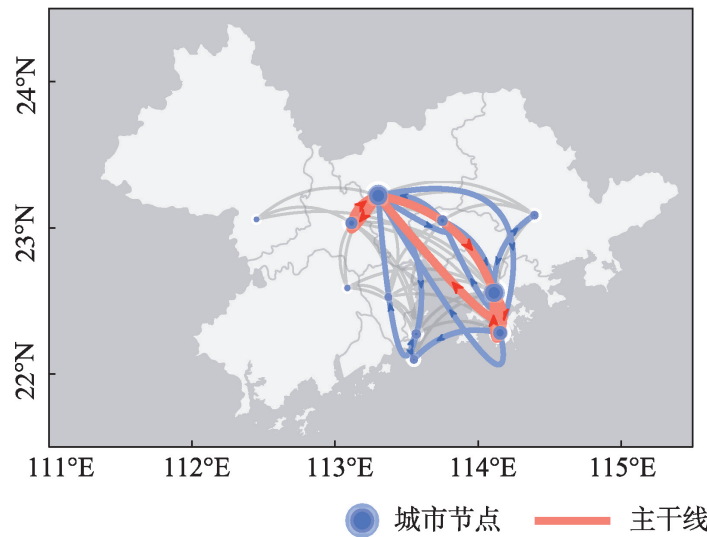

(b) 关注网络

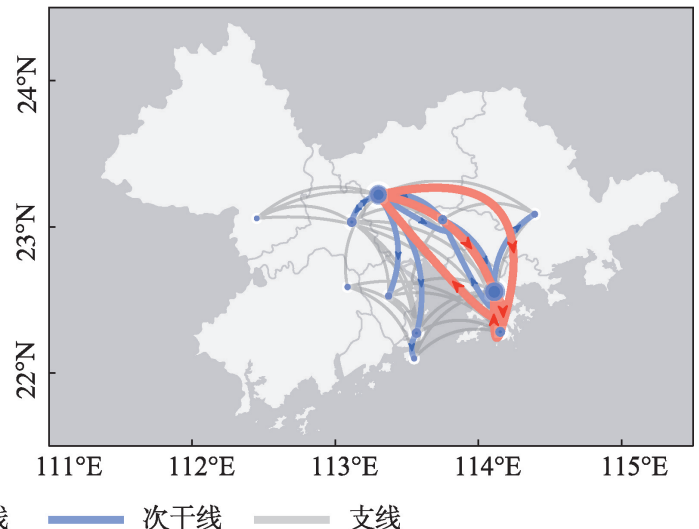

图 1 粤港澳大湾区城市网络联系

Fig.1 Connectivity of the Guangdong-Hong Kong-Macao Greater Bay Area's city network

表 2 粤港澳大湾区城市网络联系强度

Tab.2 Connection rate of the Guangdong-Hong Kong-Macao Greater Bay Area's city network

\begin{tabular}{|c|c|c|c|c|c|c|c|c|}
\hline \multirow{2}{*}{ 排序 } & \multicolumn{4}{|c|}{ 流动网络 } & \multicolumn{4}{|c|}{ 关注网络 } \\
\hline & 起点 & 终点 & 流动量 & 相对值 & 起点 & 终点 & 关注度 & 相对值 \\
\hline 1 & 深圳 & 香港 & 2976 & 1.00 & 深圳 & 香港 & 1601 & 1.00 \\
\hline 2 & 香港 & 深圳 & 2608 & 0.88 & 广州 & 深圳 & 752 & 0.47 \\
\hline 3 & 广州 & 深圳 & 2304 & 0.77 & 深圳 & 广州 & 715 & 0.45 \\
\hline 4 & 深圳 & 广州 & 2069 & 0.70 & 广州 & 香港 & 551 & 0.34 \\
\hline 5 & 佛山 & 广州 & 2003 & 0.67 & 香港 & 深圳 & 550 & 0.34 \\
\hline 6 & 广州 & 佛山 & 1976 & 0.66 & 佛山 & 广州 & 423 & 0.26 \\
\hline 7 & 东莞 & 深圳 & 999 & 0.34 & 广州 & 中山 & 390 & 0.24 \\
\hline 8 & 深圳 & 东莞 & 987 & 0.33 & 珠海 & 澳门 & 377 & 0.24 \\
\hline 9 & 广州 & 东莞 & 759 & 0.26 & 广州 & 佛山 & 302 & 0.19 \\
\hline 10 & 东莞 & 广州 & 752 & 0.25 & 东莞 & 深圳 & 281 & 0.18 \\
\hline
\end{tabular}

此外, 广州对香港的单向关注度相对值为 0.34 , 高于流动量的 0.24 , 凸显出了两地跨域信息交 流的需求; 在广深廊道的辐射带动下, 东莞的城市 节点发展初显成效, 与广深均建立了双向次级联 系, 流动量相对值介于 $0.25 \sim 0.34$, 但关注度却低于 0.18 。

\section{3 中国城市网络下的广深对比分析}

全国尺度下, 选取了 365 个网络节点,包括 334 个地级市、 4 个直辖市、港澳台及 24 个省辖县。研 究时段内共有 9970715 条国内签到微博, 从中提取 度中心性前 30 名的城市节点构建联通子图(表3、图 2)。其中, 北上广位列前 3 位, 而深圳则在流动与关 注网络中分别位于第 6 、第 4 位。

以节点特征来看(表 3 ), 交通基础设施作为跨城
表 3 广深在中国城市网络中的指标

Tab.3 Indices of Guangzhou and Shenzhen in China's city network

\begin{tabular}{cccccc}
\hline \multirow{2}{*}{ 指标 } & \multicolumn{2}{c}{ 流动网络 } & & \multicolumn{2}{c}{ 关注网络 } \\
\cline { 2 - 3 } \cline { 5 - 6 } \cline { 5 - 6 } & 广州 & 深圳 & & 广州 & 深圳 \\
\hline 度中心性 & 34830 & 30062 & & 16013 & 15820 \\
接近中心性 & 0.1659 & 0.1606 & & 0.0493 & 0.0490 \\
首位联系 & 13 & 6 & & 12 & 6 \\
次位联系 & 14 & 7 & & 12 & 12 \\
\hline
\end{tabular}

人流的空间载体,成为了塑造网络的组织轴线。流 动网络中, 广州是华南最大的综合交通枢纽, 度中 心性达 34830 , 较深圳高出 4768 ; 联系广度方面, 广 州海陆空铁网络较为成熟,首、次位联系主要位于 粤西、粤北及湘桂地区, 平均旋转半径达 $697 \mathrm{~km}$, 可 辐射至长沙、贵阳、南宁等城市, 腹地市场发展潜力 巨大。深圳融人国家铁路网络的程度、客货运量均 低于广州(广州市统计局, 2017; 深圳市统计局, 
(a) 流动联系

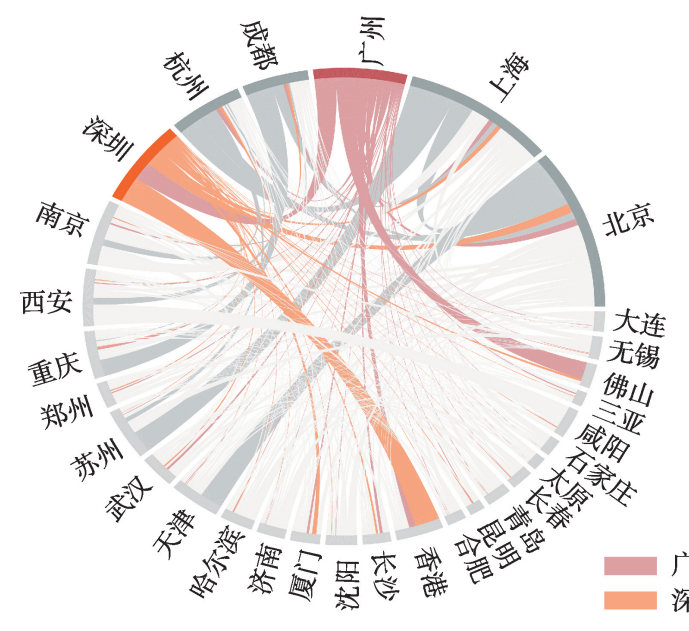

(b) 关注联系

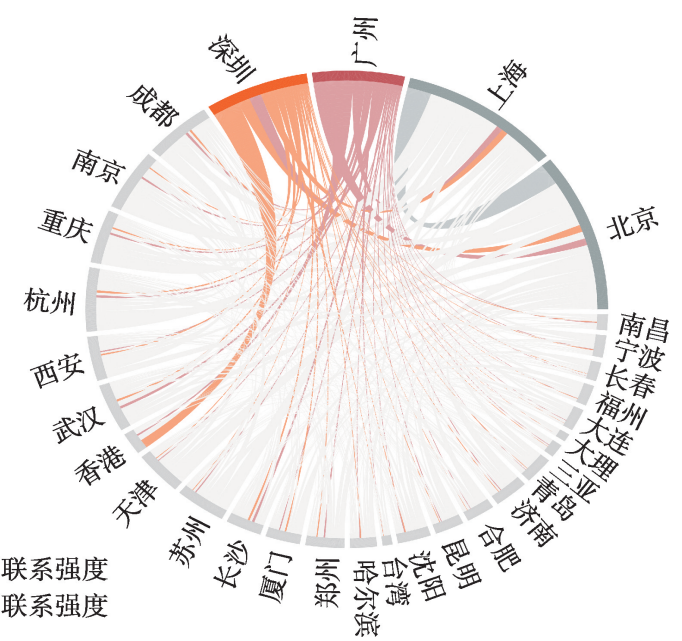

图 2 广深在中国城市网络中的和弦图

Fig.2 Chord diagram of Guangzhou and Shenzhen in China's city network

2017), 度中心性仅为 30062; 联系广度方面, 首、次 位联系主要位于粤东及闽赣地区, 平均旋转半径为 $601 \mathrm{~km}$,可覆盖厦门、福州、新余等邻近城市。

关注网络中, 与湾区网络所不同的是, 广州的 度中心性略高于深圳, 呈现出了路径依赖效应(魏 宗财等, 2013)。尽管信息流成为了新的要素组织 动力, 但广州、深圳的决策营运部门与传输媒介载 体等物化的地理支撑仍存在明显的空间分异,信息 流无法完全脱离实体空间成为塑造网络的主导力 量(杨家文, 1999)。广州作为中国 3 大通信枢纽之 一, 度中心性达 16013 , 其关注网络的演进遵循通信 设施的积累进程与流动路径的发展惯性不断自我 强化,结果符合理论预期。深圳的信息交流格局则 内嵌于产业网络, 接近中心性为 0.0490 , 网络可达
性与广州差异较小,但仍需更为广泛高效的载体布 局,以提升信息负载能力。

以联系格局来看,广深展现出了多种联系导向 (表4),其中,信息流呈现出了协同补充效应(魏宗财 等, 2008)。流动网络中, 深圳与北京、厦门联系紧 密, 流动量相对值达 0.38 以上, 广州则与上海、武 汉、杭州互动活跃,流动量相对值达 0.40 以上,但在 关注网络中,两者的联系导向却大致相反,可能的 解释是广深的实体流动联系受距离、供求等机制的 约束,而信息流部分补充了地方场所中的实际位 移,开辟了更为广泛的城市互动选择,提升了组织 要素的灵活性,缩小了区位差异。这一效应在湾区 网络中同样有所体现,例如广港之间距离较远,但 关注度相对值高于流动量；而广佛、深莞区位相邻，

表 4 广深在中国城市网络中的联系强度(过滤湾区内联系)

Tab.4 Connection rate of Guangzhou and Shenzhen in China's city network (filtering out the bay area connections)

\begin{tabular}{|c|c|c|c|c|c|c|c|c|}
\hline \multirow{2}{*}{ 排序 } & \multicolumn{4}{|c|}{ 流动网络 } & \multicolumn{4}{|c|}{ 关注网络 } \\
\hline & 起点 & 终点 & 流动量 & 相对值 & 起点 & 终点 & 关注度 & 相对值 \\
\hline 1 & 北京 & 深圳 & 1043 & 1.00 & 广州 & 北京 & 1015 & 1.00 \\
\hline 2 & 深圳 & 北京 & 943 & 0.90 & 深圳 & 北京 & 731 & 0.72 \\
\hline 3 & 北京 & 广州 & 796 & 0.76 & 深圳 & 上海 & 505 & 0.50 \\
\hline 4 & 广州 & 北京 & 774 & 0.74 & 广州 & 上海 & 493 & 0.49 \\
\hline 5 & 广州 & 上海 & 593 & 0.57 & 北京 & 深圳 & 466 & 0.46 \\
\hline 6 & 上海 & 广州 & 543 & 0.52 & 北京 & 广州 & 450 & 0.44 \\
\hline 7 & 深圳 & 上海 & 521 & 0.50 & 上海 & 广州 & 389 & 0.38 \\
\hline 8 & 上海 & 深圳 & 487 & 0.47 & 上海 & 深圳 & 384 & 0.38 \\
\hline 9 & 广州 & 杭州 & 446 & 0.43 & 广州 & 成都 & 297 & 0.29 \\
\hline 10 & 厦门 & 深圳 & 441 & 0.42 & 深圳 & 成都 & 248 & 0.24 \\
\hline
\end{tabular}


具有较高的流动量,但关注度却相对不足。

另一方面,地方场所仍具有持续效力, 信息流 存在明显的路径依赖效应(魏宗财等, 2013), 主要体 现在深圳与漳州、九江, 广州与长沙、贵阳的流动、 关注导向保持一致,且 2 类联系的相对值之差较低， 均位于 0.06 以下。由于隐性知识的传递与大多数 生产生活服务仍需面对面交流, 广深的实体流动提 升了主体互动概率与信息传播途径,而信息流又反 过来扩大了人群的空间感知, 产生了更多的出行需 求。在多重效应的叠加交融下, 传统的地缘联系差 异转向了复合型组织机制, 广深的区域联系趋向柔 性化(周玉翠等, 2006)。

此外,广、深联系格局不仅是节点体量、等级与 影响力的体现, 亦是城市联系博峦的结果, 本文运 用比值法进一步探索优势联系(曹子威等, 2013)。 若某市与广、深的联系比值介于 $0.8 \sim 1.2$ 之间, 为双 城联系均衡型; 若比值小于 0.8 , 为深圳联系优势 型; 若比值大于 1.2 , 为广州联系优势型。

流动网络中(图 3a), 广深的联系遵循距离衰减 规律, 与胡焕庸线以西的城市交互薄弱, 流动量相 对值均处于 0.07 以下, 多为联系均衡型城市; 其次, 单城优势型城市呈集聚连片分布, 基本上以京广铁 路为中轴线, 西侧主要为广州联系优势, 东侧主要 为深圳联系优势。广州充分发挥京广铁路的廊道 效应连接了 6 省省会, 并经由沪汉蓉高铁实现了与 长三角、成渝城市群的快速通达, 与 126 个城市构建 了联系优势。深圳在东北侧沿京九铁路向内陆延 伸, 但仅途经南昌 1 个省会, 区域联动辐射能力有 限, 东侧则沿厦深铁路与海峡西岸城市群紧密对
接,仅有 54 个联系优势城市。

关注网络中(图 3b), 广深的跨域性联系增强, 打 破了基于传统中心地理论的区域空间结构。广州 汇集了华南大区的邮政、央行等决策营运中心,在 行政指向下,信息流先遵循等级扩散的路径传递至 大部分省会城市,再以接触扩散向周边传播, 与 88 个城市构建了联系优势。深圳在地缘政治影响力 等方面弱于广州, 但经早期对市场经济的探索, 信 息经济渗透、实践经验推广的开放性更加显著,在 市场指向下, 深圳对各省外围地区的辐射带动相对 占优, 呈现出飞地式镶嵌的格局, 共有 57 个联系优 势城市, 数目差距有所减小, 且多为“信息腹地 $\longrightarrow$ 深 圳”的单向关注。

\section{4 全球城市网络下的广深对比分析}

全球尺度下,城市网络会在一定程度上受到微 博使用群体、社会文化背景、国家政治体制等多方 差异的影响(Hawelka et al, 2014)。因此,借鉴Son (2014)对近 $30 \mathrm{a}$ 的 22 个全球城市网络研究的统计 分析, 结合城市微博数据量与用户覆盖率, 选取了 184 个主要城市节点。需要指出的是, 鉴于香港的 国际地位及其与广深的紧密联系, 本节将其一同纳 人网络进行比较。研究时段内共有 201587 条国外 签到微博, 提取度中心性前 60 名的节点构建联通子 图(图4)。

以节点特征来看(表 5), 广州的全球流动、关注 度中心性皆高于深圳, 与全国网络表现出了一致 性。流动网络中, 香港的度中心性最高, 其次为广

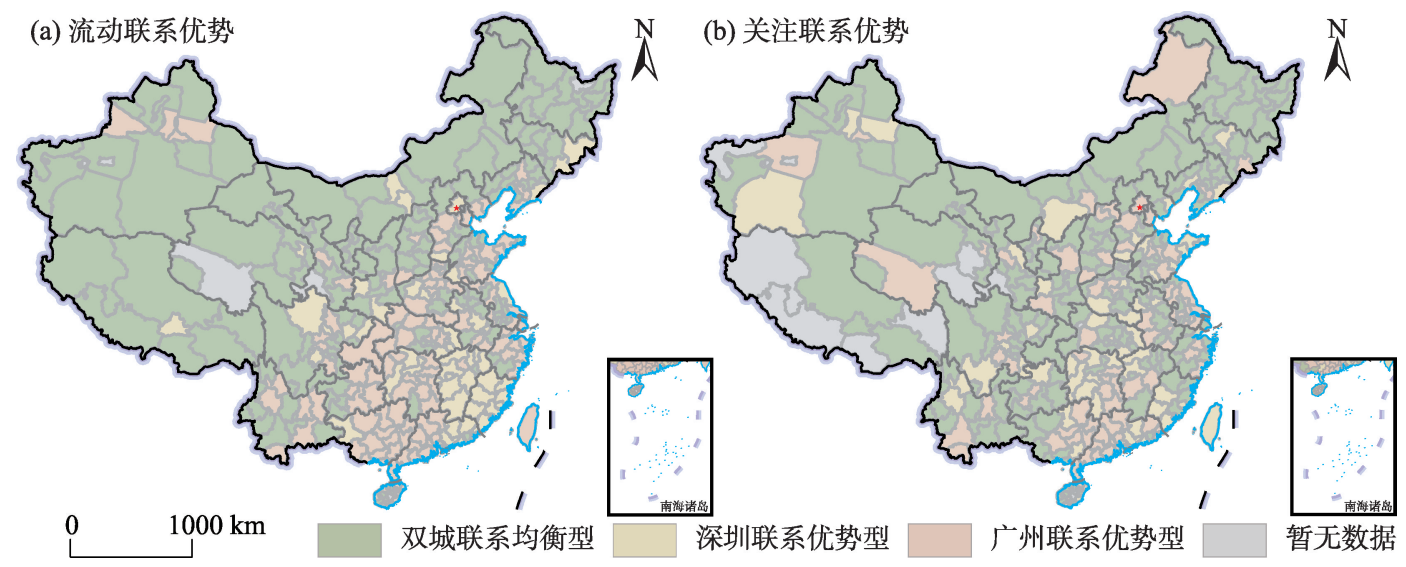

注:本图基于自然资源部标准地图服务网站下载的审图号为 GS(2019)1827号的标准地图制作,底图无修改。下同。

图 3 广深在中国城市网络中的联系优势对比

Fig.3 Comparison of prioritized connections between Guangzhou and Shenzhen in China's city network 
(a) 流动联系优势

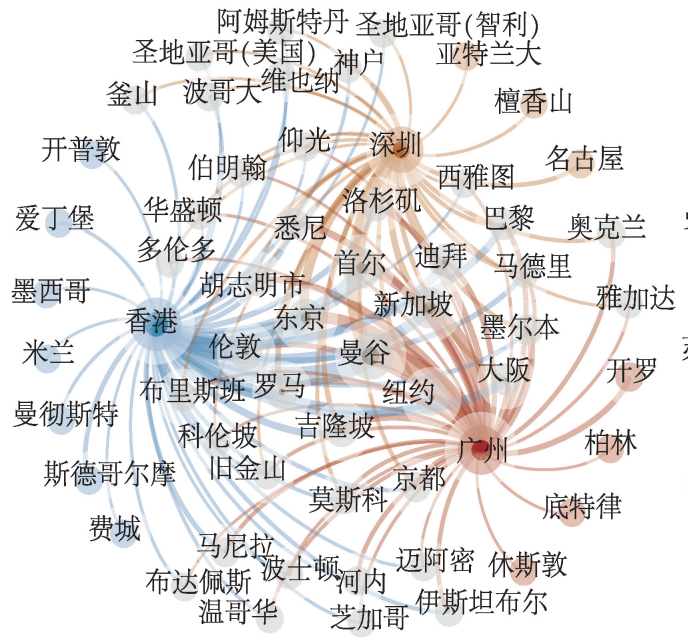

香港优势联系 深圳优势联系 (b) 关注联系优势

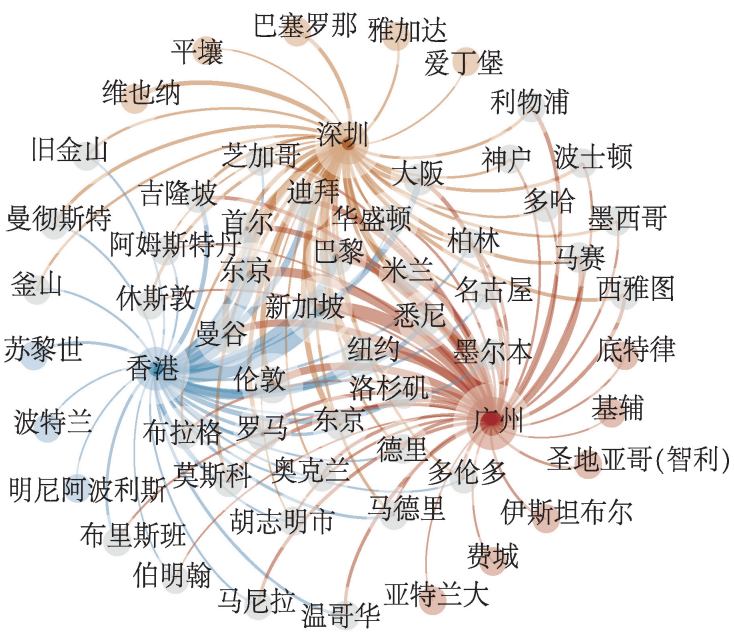

图 4 广深在全球城市网络中的联系优势对比

Fig.4 Comparison of prioritized connections between Guangzhou and Shenzhen in global city network

表 5 广深在全球城市网络中的指标

Tab.5 Indices of Guangzhou and Shenzhen in global city network

\begin{tabular}{|c|c|c|c|c|c|c|}
\hline \multirow{2}{*}{ 指标 } & \multicolumn{3}{|c|}{ 流动网络 } & \multicolumn{3}{|c|}{ 关注网络 } \\
\hline & 广州 & 深圳 & 香港 & 广州 & 深圳 & 香港 \\
\hline 度中心性 & 847 & 604 & 958 & 1054 & 935 & 990 \\
\hline 接近中心性 & 0.0170 & 0.0165 & 0.0173 & 0.0167 & 0.0164 & 0.0166 \\
\hline 首位联系 & 42 & 20 & 50 & 50 & 36 & 45 \\
\hline 次位联系 & 26 & 24 & 32 & 46 & 34 & 20 \\
\hline
\end{tabular}

州。广州通过全球粤侨关系网吸纳了大量外资, 以 “广交会”融人国际市场, 并依托白云国际空港、南 沙深水海港等战略资源打开了对外通道, 历史惯性 与交通优势塑造了其商贸枢纽的地位, 度中心性达 847 , 较深圳高出 243 ; 联系广度方面, 其平均旋转半 径达 $3185 \mathrm{~km}$,与曼谷、新加坡等城市形成了首位联 系, 未来,广州将建设成为引领全球现代交通发展 的综合枢纽。

深圳单节点的流动联系能力较弱, 度中心性仅 为 604 , 在国际航点覆盖、客运量等方面较广州仍有 差距(广州市统计局, 2017; 深圳市统计局, 2017), 平 均旋转半径为 $2870 \mathrm{~km}$; 但香港作为国际中转枢纽, 航空网络广阔, 2017 年接待旅客超 7200 万人次(刘 倩, 2018),近年来通过“经深飞”“深港互飞”等项目 的中转衔接, 有效促进了国内、国际航线的资源互 补。根据流动路径分析, 在“深一港”流动群体中, 有 $8.95 \%$ 的用户在流动链上下游与国外城市具有联 系, 而在 “广一港”流动群体中仅为 $4.08 \%$, 这说明由
于深圳国际航线的缺失, 部分国际客流需经香港中 转, 间接反映出了深圳的对外联系对香港仍有一定 的依存度。

关注网络中,信息化驱动着全球生产环节的空 间分离,塑造了“信息集中一空间分散”的联系模式 (孙中伟等, 2009)。广州度中心性为 1054 ,信息辐射 优势主要体现在信息媒介与使领馆、国际友城关系 网的支撑。作为中国三大互联网国际出人口之一, 广州的国际局电路可直达 70 多个国家(刘茜, 2017), 接近中心性达 0.0167 ,显示出了良好的信息 接人性,进而吸引全球经济活动的集聚,但也存在 创新能力不足、高端引领较弱等问题(孙莉, 2013)。

深圳关注度中心性为 935 , 略低于广州, 但在知 识共享、技术互补、成果转化等方面具有一定的制 度厚度,创新驱动效应显著,信息经济持续增长(孙 莉, 2013)。联系导向方面, 深圳与伦敦、纽约等城 市形成了首位联系, 关注度相对值介于 $0.24 \sim 0.62$ 之 间。此外,香港经由深圳向内地辐射国际市场信 
息, 两者以良性双向互动、先进制度规则引领着全 球信息的集疏,进而支撑着深圳扩大对外开放空 间、深度参与国际分工合作。

以联系格局来看(图 4a), 相较于湾区、全国网 络, 随着节点间距离的增加, 信息流的协同补充效 应更为显著, 并驱动着广深的对外联系趋向分散, 与实体人流呈现出了一定差异, 这表明了实虚网络 的动态发展具有不同的逻辑。流动网络中, 广州、 深圳的主导联系覆盖曼谷、东京、新加坡等邻近城 市, 流动量相对值介于 0.37 1 之间(表 6), 但与欧美 主要城市的流动量却不足 0.26 , 说明地理距离仍是 实体网络联系的重要影响因素。其次, 曼谷与广深 港三地均形成了紧密的联系, 流动量相对值达 0.44 以上, 反映出了部分国际签到数据可能偏向于旅游 行为的表征内涵。

另一方面, 关注网络中的主导联系则大幅改变 (图 4b), 广州、深圳与纽约、伦敦、巴黎的关注联系跃 居前列, 相对值介于 $0.34 \sim 0.62$ 之间(表 6), 部分城市 功能交互趋向虚拟化、信息化,成为了新的网络联 系支撑。此外, 在 $10000 、 20000 、 30000 \mathrm{~km}$ 三个圈层 中, 关注度分别占 2 类联系总和的 $46.08 \% 、 58.43 \%$ 、 $68.13 \%$, 该结果指出了信息流可能在广深的远距离 互动中发挥着更加关键的作用, 通过区域外包、离 岸业务、技术转移等信息密集型手段, 重塑了广深 的对外联系格局,缩短了全球网络间的感知距离。

\section{5 结论与展望}

本文基于微博数据, 运用社会网络分析等方 法, 辨析了广州、深圳在多尺度下流动、关注网络的
联系差异,为城市网络、“流空间”理论提供了新的 视角。研究得出以下结论:

(1) 多尺度网络下,广州、深圳不同的发展路径 与联系导向塑造了差异化的要素组织能力, 同时也 存在距离、资源、供求等约束机制。其联系特征为: (1) 湾区尺度下, 广州倾向建立均衡联系, 深圳则相 对集中; (2) 全国尺度下,广州占据联系枢纽,快速融 人沿线走廊, 深圳则因联系通道受限, 主要辐射闽 赣地区; 3 全球尺度下,广州依托商贸发展与基础 设施优势打开了对外通道, 深圳则与香港联系尤为 紧密。

（2）实虚网络之间,广州、深圳表现出了多种联 系导向,且在各尺度下具有不同的发展与体现。一 方面,信息流遵循物化载体的空间布局与实体流动 的发展惯性不断强化，具有显著的路径依赖效应; 另一方面, 信息流作为实体流动的补充, 拓展了人 们的空间感知, 促使部分城市功能联系趋向信息 化, 展现出了一定的协同补充效应。多重效应的叠 加交融提升了网络的广度与深度, 推动着城市网络 联系趋向柔性化。

（3）基于上述的城市网络格局,广州、深圳应立 足于不同的联系模式与发展实际, 引领打造有序高 效、协同联通的城市网络系统。(1) 在湾区内部, 广 州、深圳应坚持极点带动、辐射周边的发展战略, 一 方面以交通、信息基础设施互联互通为基础,逐步 发挥产业梯度转移效益, 引领湾区城市全面务实合 作、协同联动发展; 另一方面, 以社会民生联系为纽 带, 加强与港澳的高效衔接与体制协调, 创新通关 模式、提升通关能力,进而在人才流动、信息集散与 商贸合作等方面寻求更大的成长空间。(2)在全国

表 6 广深在全球城市网络中的联系强度(过滤国内联系)

Tab.6 Connection rate of Guangzhou and Shenzhen in global city network (filtering out domestic connections)

\begin{tabular}{|c|c|c|c|c|c|c|c|c|}
\hline \multirow{2}{*}{ 排序 } & \multicolumn{4}{|c|}{ 流动网络 } & \multicolumn{4}{|c|}{ 关注网络 } \\
\hline & 起点 & 终点 & 流动量 & 相对值 & 起点 & 终点 & 关注度 & 相对值 \\
\hline 1 & 广州 & 曼谷 & 83 & 1.00 & 广州 & 新加坡 & 94 & 1.00 \\
\hline 2 & 香港 & 曼谷 & 75 & 0.90 & 广州 & 东京 & 73 & 0.78 \\
\hline 3 & 香港 & 东京 & 59 & 0.71 & 深圳 & 伦敦 & 58 & 0.62 \\
\hline 4 & 曼谷 & 广州 & 55 & 0.66 & 深圳 & 纽约 & 55 & 0.59 \\
\hline 5 & 首尔 & 香港 & 53 & 0.64 & 新加坡 & 香港 & 51 & 0.54 \\
\hline 6 & 深圳 & 曼谷 & 49 & 0.59 & 深圳 & 巴黎 & 50 & 0.53 \\
\hline 7 & 广州 & 新加坡 & 46 & 0.55 & 深圳 & 新加坡 & 49 & 0.52 \\
\hline 8 & 曼谷 & 香港 & 45 & 0.54 & 广州 & 纽约 & 48 & 0.51 \\
\hline 9 & 东京 & 香港 & 43 & 0.52 & 香港 & 新加坡 & 47 & 0.50 \\
\hline 10 & 香港 & 首尔 & 40 & 0.48 & 香港 & 东京 & 46 & 0.49 \\
\hline
\end{tabular}


网络中, 湾区作为经济增长的重要引擎, 上升为国 家战略后, 更应成为全国有引领作用的创新中心。 广州、深圳应坚持轴带支撑、创新驱动的发展战略, 构建运作高效、衔接顺畅的对外联系通道, 塑造区 域经济发展轴线, 深化湾区与海峡西岸城市群、中 南地区、长江中游地区、西南地区的交流合作, 实现 基础设施网络、信息流通渠道、城市功能资源的相 互支持与融通促进, 形成全国的创新高地。(3) 在国 际联系上,湾区应服从国家战略发展大局, 因而广 州、深圳应以开放包容的姿态建立与全球城市间的 联系互动, 更深广地融人全球经济体系, 成为“一带 一路”倡议的重要支撑区域; 另一方面, 面对世界三 大湾区的竞争, 应推进广州-深圳国际性综合交通 枢纽建设, 提升互联网流量转接能力, 建立与国际 接轨的开放型经济制度、法律体系, 开拓意识形态 话语的全球视野,建成高水平的对外开放门户。

不可否认的是, 微博数据存在内生性的用户群 体偏差、地区用户覆盖率差异等局限, 还需补充其 他 “流数据” 展开验证与探讨, 以提升研究结果的稳 健性; 其次, 未来研究可考虑通过网络时序变化的 视角, 全面刻画城市网络的关联动态与发展趋向; 此外, 网络中所展现出的政策壁垒、制度差异对要 素流动的影响机制, 尚待进一步深人探究。

\section{参考文献(References)}

曹子威, 罗震东, 耿否. 2013. 基于信息流的城市-区域关系 比较研究: 以马鞍山和芜湖为例 [J]. 经济地理, 33(5): 4753. [Cao Z W, Luo Z D, Geng L. 2013. Information-flow based comparative research of urban- region relations: A case study of Ma'anshan and Wuhu. Economic Geography, 33(5): 47-53. ]

董超. 2012. “流空间” 的地理学属性及其区域发展效应分析 [J]. 地域研究与开发, 31(2): 5-8. [Dong C. 2012. On the geographical attributes of space of flows and their effects in regional development. Areal Research and Development, 31(2): 5-8. ]

广州市统计局. 2017. 广州统计年鉴(2017) [M]. 北京: 中国 统计出版社. [Guangzhou Statistics Bureau. 2017. Guangzhou Statistical Yearbook (2017). Beijing, China: China Statistics Press. ]

柯文前, 俞肇元, 陈伟, 等. 2015 . 人类时空间行为数据观测 体系架构及其关键问题 [J]. 地理研究, 34(2): 373-383.

[Ke W Q, Yu Z Y, Chen W, et al. 2015. Architecture and key issues for human space- time behavior data observation. Geographical Research, 34(2): 373-383. ] 林雄斌, 杨家文, 林倩. 2015. 都市区中心城与次区域跨界协
调发展探讨: 以宁波为例 [J]. 城市观察, (4): 74-86. [Lin X B, Yang J W, Lin Q. 2015. An exploration of cross-border governance and coordinated development between the central city and subregions in metropolitan areas: A case study of Ningbo. Urban Insight, (4): 74-86. ]

刘茜. 2017. 广州具备坚实基础 建设国际科技创新枢纽[N/ OL]. 南方日报, 2017-07-07(GC03). http://epaper.southen. com/nfdaily/html/2017-07/07/content_7651136.htm. [Liu Q. 2017. Guangzhou has a solid foundation to build an international technological innovation hub. Nanfang Daily, 201707- 07(GC03). http://epaper.southcn.com/nfdaily/html/201707/07/content_7651136.htm. ]

刘倩. 2018. 粤港澳大湾区民航需求强劲 [N/OL]. 南方日报, 2018-03-29(A12). http://epaper.southcn.com/nfdaily/html/ 2018-03/29/content_7712672.htm. [Liu Q. 2018. Civil aviation is demanded strongly in Guangdong- Hong KongMacao Greater Bay Area. 2018-03-29(A12). http://epaper. southen.com/nfdaily/html/2018-03/29/content_7712672.htm. ] 聂晶金金, 黄亚平, 刘合林, 等. 2017. 基于社会网络分析的武 汉城市圈城镇生活性关联特征 [J]. 经济地理, 37(3): 6370. [Nie J X, Huang Y P, Liu H L, et al. 2017. The associated features of urban life in Wuhan metropolitan area based on social network analysis. Economic Geography, 37(3): 63-70. ]

深圳市统计局. 2017. 深圳统计年鉴(2017) [M]. 北京: 中国 统计出版社. [Shenzhen Statistics Bureau. 2017. Shenzhen Statistical Yearbook (2017). Beijing, China: China Statistics Press. ]

孙莉. 2013. 广州与深圳城市创新职能比较研究 [D]. 广州: 广州大学. [Sun L. 2013. Comparative study on development force in underdeveloped areas of Guangdong. Guangzhou, China: Guangzhou University. ]

孙中伟, 路紫, 贺军亮. 2009. 世界互联网信息流的空间格局 及其组织机理 $[\mathrm{J}]$. 人文地理, 24(4): 43-49. [Sun Z W, Lu Z, He J L. 2009. Research on spatial pattern and organization mechanism of international internet information flows. Human Geography, 24(4): 43-49. ]

王姣娥, 景悦. 2017. 中国城市网络等级结构特征及组织模 式: 基于铁路和航空流的比较 [J]. 地理学报, 72(8): 15081519. [Wang J E, Jing Y. 2017. Comparison of spatial structure and organization mode of inter- city networks from the perspective of railway and air passenger flow. Acta Geographica Sinica, 72(8): 1508-1519. ]

王萌, 匡耀求, 黄宁生. 2017. 珠江三角洲城际间人口流动倾 向空间特征: 基于网络关注度数据的时空演化 [J]. 热带 地理, 37(1): 33-42. [Wang M, Kuang Y Q, Huang N S. 2017. Spatial characteristics of intercity population flow propensity in the Pearl River Delta region: Based on the 
spatio-temporal change of internet attention index. Tropical Geography, 37(1): 33-42. ]

王永明, 马耀峰, 王美霞. 2012. 中国人境游客多城市旅游空 间网络结构 [J]. 地理科学进展, 31(4): 518-526. [Wang Y M, Ma Y F, Wang M X. 2012. Network structure of multicity inbound tourists to China. Progress in Geography, 31 (4): 518-526. ]

魏宗财, 甄峰, 席广亮, 等. 2013. 全球化、柔性化、复合化、差 异化: 信息时代城市功能演变研究 [J]. 经济地理, 33(6): 48-52. [Wei Z C, Zhen F, Xi G L, et al. 2013. Globalization, flexibility, composition, differentiation: Study on the evolution of urban functions in the information age. Economic Geography, 33(6): 48-52. ]

魏宗财, 甄峰, 张年国, 等. 2008. 信息化影响下经济发达地 区个人联系网络演变: 以苏锡常地区为例 [J]. 地理科学 进展, 27(4): 82-88. [Wei Z C, Zhen F, Zhang N G, et al. 2008. The evolution characteristics of personal communication network of economically developed regions under the influence of informationization: A case study of Suzhou-Wuxi-Changzhou area. Progress in Geography, 27 (4): 82-88. ]

谢永俊, 彭霞, 黄舟, 等. 2017. 基于微博数据的北京市热点 区域意象感知 [J]. 地理科学进展, 36(9): 1099-1110. [Xie Y J, Peng X, Huang Z, et al. 2017. Image perception of Beijing's regional hotspots based on Microblog data. Progress in Geography, 36(9): 1099-1110. ]

杨家文. 1999. 信息时代城市结构变迁的思考 [J]. 城市发展 研究, (4): 15-18. [Yang J W. 1999. Pondering on urban structural transformation in the communication era. Urban Studies, (4): 15-18. ]

姚永玲, 唐彦哲. 2015. 城市群首位城市的联系能级、中心度 和控制力 [J]. 经济地理, 35(7): 66-71. [Yao Y L, Tang Y Z. 2015. Inter connection, centrality and power of primate city within city cluster. Economic Geography, 35(7): 66-71. ] 张林, 刘继生. 2006. 信息时代区位论发展的新趋势 [J]. 经济 地理, 26(2): 181-185. [Zhang L, Liu J S. 2006. New tendency of location theory in information age. Economic Geography, 26(2): 181-185. ]
周玉翠, 陆玉麒, 陈晓华, 等. 2006. 网络化时代中部崛起新 思维 [J]. 人文地理, 21(2): 124-128. [Zhou Y C, Lu Y Q, Chen X H, et al. 2006. New idea on rising of central region in China in network era. Human Geography, 21(2): 124128. ]

Alderson A S, Beckfield J. 2004. Power and position in the world city system [J]. American Journal of Sociology, 109 (4): 811-851.

Belyi A, Bojic I, Sobolevsky S, et al. 2017. Global multi-layer network of human mobility [J]. International Journal of Geographical Information Science, 31(7): 1381-1402.

Cairncross F. 2001. The death of distance: How the communications revolution is changing [M]. Cambridge, USA: Harvard Business School.

Castells M. 1996. The rise of the network society: The information age: Economy, society, and culture [M]. Malden, USA: Blackwell.

Choi J H, Barnett G A, Chon B S. 2006. Comparing world city networks: A network analysis of internet backbone and air transport intercity linkages [J]. Global Networks, 6(1): 81-99.

Friedmann J. 1986. The world city hypothesis [J]. Development and Change, 17(1): 69-83.

Hawelka B, Sitko I, Beinat E, et al. 2014. Geo-located Twitter as proxy for global mobility patterns [J]. Cartography and Geographic Information Science, 41(3): 260-271.

Sassen S. 1991. Global city [M]. Princeton, USA: Princeton University Press.

Son J S. 2014. Hyperlink network system and image of global cities: Webpages and their contents [D]. Charlotte, USA: University of North Carolina.

Xu Y, Belyi A, Bojic I, et al. 2018. Human mobility and socioeconomic status: Analysis of Singapore and Boston [J]. Computers, Environment and Urban Systems, 72: 51-67.

Yin J, Soliman A, Yin D, et al. 2017. Depicting urban boundaries from a mobility network of spatial interactions: A case study of Great Britain with geo-located Twitter data [J]. International Journal of Geographical Information Science, 31(7): 1293-1313. 


\title{
City network by mobility and attention indices: A comparison of Guangzhou and Shenzhen
}

\author{
WU Xuan, YANG Jiawen* \\ (School of Urban Planning and Design, Peking University Shenzhen Graduate School, Shenzhen 518055, Guangdong, China)
}

\begin{abstract}
City networks have experienced rapid reconstruction in the past decades due to the development of city-regions. In the Guangdong-Hong Kong-Macao Greater Bay Area, Guangzhou and Shenzhen are two pivotal cities. They play key roles in promoting regional development. Therefore, it is of great significance to identify their influence areas, which can inform urban management and regional planning. Meanwhile, increasing availability of social media data creates opportunities for relevant research. The pervasive presence of locationbased services and the associated content make it possible for researchers to gain an unprecedented access to the direct records of human activities and perceptions. Much of existing literature, however, pays little attention to the differences in multi-scale network or to the relationship between the real-world network and virtual network, which are both presented in datasets of this kind. Our research contributes to the literature in both the methodological and the empirical aspects. First, we investigated the node and link characteristics of the influence areas of Guangzhou and Shenzhen by computing social network indicators with a dataset of almost 10 million Sina Microblog records between January 1 and February 6, 2018. Indices of mobility and attention were computed based on characteristics such as consecutive locations, degree centrality, closeness centrality, and average radius of gyration. These indices help to catch the interaction between real and virtual networks. Second, in order to understand inter-city mobility and attention characteristics of Guangzhou and Shenzhen, we mapped city networks of multi-scale, where edge weights denote interaction strengths. Third, our analysis confirmed that the Sina Microblog data exhibit similar statistical properties as other city network datasets. Based on the result of analyses, we argue that Guangzhou had more balanced influence in various directions, representing efficiency in hinterland connection and resource integration. Shenzhen's area of influence was relatively concentrated, with a strong tie with neighboring Hong Kong. Overall, Guangzhou competes better in the mobility network while Shenzhen competes better in the attention network. A complementary relationship was also identified between those two networks. In conclusion, we propose that Guangzhou and Shenzhen took advantage of their respective role as the hubs of regional transportation and innovation as well as what they have already accumulated and their connections with other parts of the world. They should help to build a coordinated and competitive Guangdong-Hong Kong-Macao Greater Bay Area. Our research results offer some insights for policymakers to interpret the geographic dynamics and make relevant decisions in this region. It also provides some references and inputs for analyzing social media data for the research community.
\end{abstract}

Keywords: city network; Microblog; mobility index; attention index; Guangdong-Hong Kong-Macao Greater Bay Area 